\title{
Laboratory Test Reference Identifier
}

National Cancer Institute

\section{Source}

National Cancer Institute. Laboratory Test Reference Identifier. NCI Thesaurus. Code C83116.

A sequence of characters used to identify, name, or characterize the laboratory test reference. 